\title{
Virtual-MIMO feedback and transmission for wireless ad hoc networks
}

\author{
Sungyoon Cho ${ }^{1}$, Byoung-Yoon Min ${ }^{1}$, Kaibin Huang ${ }^{2}$ and Dong Ku Kim*
}

\begin{abstract}
In a wireless ad hoc network, single-antenna nodes can cooperate in their transmissions to create a virtual multipleinput multiple-output (MIMO) channel for maximizing the spatial multiplexing gain. However, implementing virtualMIMO transmission can result in overwhelming overhead, such as channel state information (CSI) feedback and local data exchange, as the number of cooperating nodes increases. To suppress this overhead, we apply the principle of virtual-MIMO transmission to CSI feedback and design a novel framework for supporting parallel feedback streams, called virtual-MIMO feedback. Building on this feedback framework, we optimize virtual-MIMO transmission techniques and quantify their throughput gains in the presence of CSI and local-exchange overhead. To be specific, the outage capacities of these virtual-MIMO techniques are analyzed, and the corresponding bandwidth allocation for local data exchange and long-range transmission is optimized in a way to maximize overall spectral efficiency. Finally, the improvement of spectral efficiency in proposed virtual-MIMO feedback and transmission is demonstrated by the simulation results.
\end{abstract}

Keywords: Virtual MIMO, CSI feedback, Clustering

\section{Introduction}

The multiple-input multiple-output (MIMO) technology was a breakthrough in wireless communication as it improved the capacity of wireless system without increasing bandwidth and transmission power [1]. In single-user MIMO system, the spatial degrees of freedom (DoF) have been utilized to attain diversity and array gains by either maximum ratio combining (MRC) or space-time codes [2]. Moreover, MIMO techniques have been developed to achieve the full multiplexing gain in multi-user system by using space division multiple access (SDMA) schemes [3]. In practice, linear precoding schemes such as zeroforcing (ZF) and block diagonalization have been studied in imperfect channel state information (CSI) at transmitters and their matching scheduling algorithms achieving multi-user diversity gain have been proposed in [4-7]. Also, various existing studies in MIMO systems have been considered where a transmitter acquires CSI from receivers through the finite rate feedback by quantizing the estimated channel vectors; these studies show that the

*Correspondence: dkkim@yonsei.ac.kr

'School of Electrical and Electronics Engineering, Yonsei University, Seoul, South Korea

Full list of author information is available at the end of the article required number of feedback bits for each channel vector should be scaled with the number of antennas and transmit power to compensate for the rate loss due to limited feedback [4].

In modern cellular systems such as LTE-advanced, cooperative MIMO transmission schemes have been proposed to mitigate the other-cell interference in cellular networks. By allowing that the neighboring base stations (BSs) share both user data and CSI via high-capacity backhaul links, cooperative MIMO creates a multi-user MIMO system with one BS equipped with a large number of antennas, called network MIMO or coordinated multi-point transmission and reception (CoMP) in Third Generation Partnership Project (3GPP) standardization. In network MIMO, a joint transmission between BSs is performed to send the desired data to the corresponding users, which provides a considerable performance gain in multi-cell interference channels $[8,9]$. However, the network MIMO requires a huge amount of CSI feedback for all direct and interfering links as well as the desired data exchange through the backhaul links between BSs, which makes practical implementation difficult.

The cooperative MIMO system in a cellular network has been explored in the context of collaboration between 
single-antenna nodes in order to exploit spatial DoF in wireless ad hoc networks, called virtual-MIMO (VMIMO). In [10, 11], a single-source node broadcasts its desired signal to neighboring nodes in the same cluster with which they form the space-time block coding (STBC) towards its corresponding destination node, which achieves the diversity gain in the distributed network. Similar studies in $[12,13]$ show that the cooperative beamforming provides the power gain at the desired receiver as well as interference avoidance at the unintended receivers. Recently, the efficient clustering algorithm with neighboring nodes for constructing V-MIMO and the design of cooperative precoding that maximizes data rate are proposed in $[14,15]$. However, these existing studies of V-MIMO are limited to single-source to singledestination link and employ the local-exchange overhead that linearly increases with the number of cooperative nodes [10-15].

In an effort to improve the spectral efficiency in VMIMO systems, the optimal channel and power allocation between local and long-range transmission between transmitter and receiver clusters was proposed to minimize the outage capacity in [16]. In [17, 18], algorithms for efficient local data exchange were proposed, where multiple sources broadcast their desired signals over the same spectrum with simple signaling and perform the corresponding beamforming that maximizes the system performance. However, previous literature showed that CSI feedback of all channel links between receiver cluster and transmitter cluster are required to realize the cooperative beamforming, which significantly degrades the spectral efficiency as the number of nodes participating in the cooperation increases.

To overcome a huge amount of feedback overhead in the cooperative transmission scheme, this paper targets the implementation of a V-MIMO system that achieves the full multiplexing gain for a given number of existing nodes and proposes a V-MIMO feedback that supports multistream transmission to increase the accuracy of CSI at transmitters; this has not been addressed yet in the previous literature to the best of authors' knowledge. Moreover, the optimal resource allocation for cooperative transmission is analyzed to maximize the spectral efficiency with a given local-exchange overhead and the feedback channel resources. The contributions of this paper are summarized as follows.

- V-MIMO technique is proposed for CSI feedback, where the receivers simultaneously transmit their CSIs to the associated transmitters, which are cooperatively decoded at the transmitter cluster. The proposed feedback scheme provides a significant increase of total feedback bits by exploiting the spatial multiplexing gain over the orthogonal feedback channel access of traditional MIMO system which separately allocates the feedback channel-use to each receiver $[4,5]$. Based on V-MIMO feedback, we analyze the optimal resource partitioning between V-MIMO feedback and data transmission channels that maximizes the spectral efficiency of cooperative ZF beamforming in a distributed ad hoc network. As the proposed V-MIMO feedback method improves feedback channel capacity, it provides the increase of achievable MIMO transmission rate under optimal resource partitioning.

- In the presence of CSI feedback and local data exchange overhead, we analyze the outage capacity of V-MIMO transmission due to the discrepancy between local-exchange and long-range data transmission channel quality. To meet the outage capacity, the optimal number of V-MIMO nodes that maximizes the spectral efficiency is derived as a function of the distance between nodes, transmit power, and target data rate between transmitter and the corresponding receiver.

The remainder of this paper is organized as follows. The system model is introduced in Section 2, and the cooperative CSI feedback using V-MIMO is described in Section 3. In Section 4, the cooperative ZF transmission is proposed and the corresponding performance gain and the resource optimization between local data exchange and long-range transmission are analyzed in a wireless ad hoc network. Section 5 provides simulation results, and the concluding remarks are followed in Section 6.

Notation: Bold upper case and lower case letters denote matrices and vectors, respectively. $(\cdot)^{T},(\cdot)^{\dagger}$, and $\|\cdot\|$ represent the transpose, Hermitian transpose, and the Frobenius norm of a matrix, respectively. $\Gamma(a, x)=$ $\int_{x}^{\infty} t^{\alpha-1} e^{-t} d t$ is the complementary incomplete gamma function. $\mathbb{E}\{\cdot\}$ and $Q(x)$ denote the expectation over the random channel realizations and Q-function of $x$, respectively.

\section{System model}

In this paper, we consider a wireless ad hoc network comprising $M$ transmitters communicating with $M$ corresponding receivers, where each of all transmitters and receivers is equipped with a single antenna and the distance of the receivers from the corresponding transmitters is much larger than the distance between transmitters as illustrated in Fig. 1. For this scenario, we adopt the VMIMO scheme that transmitters cooperate to perform ZF joint precoding, called cooperative $Z F$ precoding for supporting $M$ data streams, without requiring cooperation between receivers that causes a significant complexity on the implementation. Under the frequency-division multiplexing (FDM) system, let $\mathbf{h}_{i}$ denote the $M \times 1$ forward 

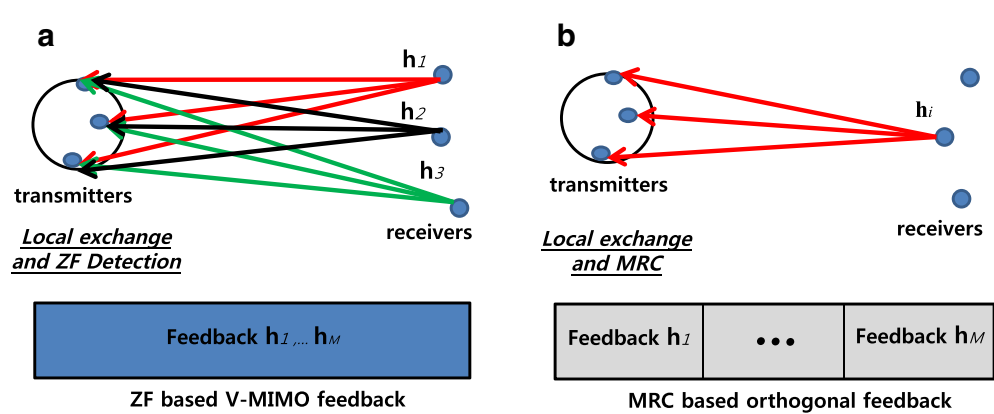

Fig. 1 Virtual-MIMO feedback scheme with transmitter cooperation. a ZF-based V-MIMO feedback and b MRC-based orthogonal feedback

channel vector from the $i$ th transmitter to all receivers and $\mathbf{g}_{i}$ denote the $M \times 1$ reverse channel vector from $i$ th receiver to all transmitters. All channel coefficients are modeled as independent and identically distributed (i.i.d.) random variables following $\mathcal{C N}(0,1)$ corresponding to rich scattering, and the relevant channels are perfectly estimated at both transmitters and receivers. Note that the distances between transmitters are relatively small to ensure that the direct line-of-sight (LOS) path shows stronger power contribution than the other non line-ofsight (NLOS) path due to the scattering factors [19], where the local channels among transmitters are considered to be a dominant LoS path in a short propagation distance, denoted as $d_{l}$. It follows that the capacity between transmitters is given as

$$
C_{\text {local }}=\log _{2}\left(1+\left|h_{R}\right|^{2} \frac{P_{l}}{N_{o}}\right)
$$

where $P_{l}=P_{T} d_{l}^{-\alpha}, P_{T}$ is the transmission power at each transmitter, $N_{o}$ is noise spectral density, and $\alpha$ is the path-loss exponent. Rician channel is represented as $h_{R}=\sqrt{\frac{K}{K+1}} h_{\mathrm{LOS}}+\sqrt{\frac{1}{K+1}} h_{\mathrm{NLOS}}$, where $h_{\mathrm{LOS}}$ is the fixed channel with unit norm and $h_{\mathrm{NLOS}}$ follows $\mathcal{C N}(0,1)$ under the assumption of $K \gg 1$. Over the high-speed local links, transmitters firstly exchange their data by frequencydivision multiple access (FDMA) and then transmit the corresponding shared data through the cooperative $\mathrm{ZF}$ precoding after CSI feedback is completed. The joint precoder $\mathbf{V}=\left[\mathbf{v}_{1}, \mathbf{v}_{2}, \cdots, \mathbf{v}_{M}\right]$ is equal to the matrix $\mathbf{H}^{\dagger}\left(\mathbf{H H}^{\dagger}\right)^{-1}$ with columns normalized to have unit norm where $\mathbf{H}=\left[\mathbf{h}_{1}, \cdots, \mathbf{h}_{M}\right]$ [20]. Assuming perfect CSI for all transmitters, the throughput for the $i$ th transmitterreceiver link is represented as

$$
R_{\mathrm{ZF}}^{i}=\log _{2}\left(1+\frac{P_{o}}{M}\left|\mathbf{h}_{i}^{T} \mathbf{v}_{i}\right|^{2}\right)
$$

where $P_{o}=P_{T} d_{o}^{-\alpha}$ and $d_{o}$ is the distance between transmitters and receivers, satisfying $\mathbf{h}_{i}^{T} \mathbf{v}_{j}=0, i \neq j$.
However, there exists residual cross-link interference in limited CSI feedback channel and the corresponding throughput is denoted by

$$
\hat{R}_{\mathrm{ZF}}^{i}=\log _{2}\left(1+\frac{P_{o} / M\left|\mathbf{h}_{i}^{T} \hat{\mathbf{v}}_{i}\right|^{2}}{\left(1+P_{o} / M \sum_{i \neq j}\left|\mathbf{h}_{i}^{T} \hat{\mathbf{v}}_{j}\right|^{2}\right)}\right)
$$

where $\hat{\mathbf{v}}_{i}$ is the normalized $i$-th column vector of $\hat{\mathbf{H}}^{\dagger}\left(\hat{\mathbf{H}} \hat{\mathbf{H}}^{\dagger}\right)^{-1}$ given $\hat{\mathbf{H}}=\left[\hat{\mathbf{h}}_{1}, \ldots, \hat{\mathbf{h}}_{M}\right]$ and $\hat{\mathbf{h}}_{i}$ is quantized by a $B$-bit random codebook that minimizes the chordal distance, for analytical simplicity ${ }^{1}$. The residual interference is bounded as $\mathbb{E}\left[\left|\mathbf{h}_{i} \hat{\mathbf{v}}_{j}\right|^{2}\right] \leq 2^{-\frac{B}{M-1}}[4,5]$.

\section{Virtual-MIMO feedback}

Note that total available bandwidth $W$ is partitioned into $W_{f}$ for CSI feedback and $W_{d}$ for data transmission to implement V-MIMO transmission, where $W_{f}+W_{d}=$ $W$. In this section, we propose a novel method of realizing collaborative spatial CSI feedback channels, called V-MIMO feedback. V-MIMO feedback allows the system bandwidth to be more efficiently partitioned and utilized for data transmission and feedback as well.

\subsection{Virtual-MIMO feedback: algorithm and performance}

To achieve the V-MIMO feedback scheme as illustrated in Fig. 1, each of $M$ receivers simultaneously transmits their estimated channel vectors $\left\{\mathbf{h}_{1}, \ldots, \mathbf{h}_{M}\right\}$ to the transmitters, where the $M$ feedback streams are jointly detected by the cooperative ZF filter over whole feedback channel. To implement the cooperative ZF filter at transmitter-side, the transmitters are required to exchange the received feedback signal and the CSI of the reverse channels $\mathbf{G}=$ $\left[\mathbf{g}_{1}, \ldots, \mathbf{g}_{M}\right]$, where $\mathbf{g}_{i}$ is the channel from the $i$ th receiver to all transmitters, which incurs relatively small overhead of channel exchange due to the high-speed intra-cluster links. Moreover, the reverse channel $\mathbf{G}$ can be estimated at the corresponding transmitters using uplink sounding signals in FDD. For V-MIMO feedback, the average feedback bits of $\mathbf{h}_{i}$ per unit time can be written as [21] 


$$
\begin{aligned}
B_{\mathrm{ZF}}^{i} & =W_{f} \cdot \mathbb{E}\left[\log _{2}\left(1+\frac{\bar{P}_{o}}{M}\left|\left(\overline{\mathbf{r}}_{\mathrm{ZF}}^{i}\right)^{\dagger} \mathbf{g}_{i}\right|^{2}\right)\right] \\
& =W_{f} \cdot \log _{2}(e) e^{1 / \frac{\bar{P}_{o}}{M}} \Gamma\left(0, \frac{M}{\bar{P}_{o}}\right) \\
& \triangleq W_{f} \cdot R_{m}
\end{aligned}
$$

where $\bar{P}_{o}=P_{R} d_{o}{ }^{-\alpha}, P_{R}$ is the transmission power at receiver cluster and $\left(\overline{\mathbf{r}}_{\mathrm{ZF}}^{i}\right)^{\dagger}$ is the $i$ th row vector of $\overline{\mathbf{R}}_{\mathrm{ZF}}=$ $\left(\mathbf{G}^{\dagger} \mathbf{G}\right)^{-1} \mathbf{G}$. For simplicity, we denote that the expected achievable rate of feedback channel between the $i$ th receiver and transmitters as $R_{m}=\log _{2}(e) e^{1 / \bar{P}_{o}} \bar{M} \Gamma\left(0, \frac{M}{\bar{P}_{o}}\right)$.

As an alternative V-MIMO feedback scheme, we consider MRC based orthogonal feedback scheme over each of $M$ orthogonal feedback channel resource. By allowing local exchange of the received feedback signals and performing MRC at transmitters, the MRC filter to decode CSI of $\mathbf{h}_{i}$ is determined as $\left(\overline{\mathbf{r}}_{\text {MRC }}^{i}\right)^{\dagger}=\mathbf{g}_{i}^{\dagger} /\left\|\mathbf{g}_{i}\right\|$ and then the average feedback bits for $\mathbf{h}_{i}$ are given by [21]

$$
\begin{aligned}
B_{\mathrm{MRC}}^{i} & =\left(W_{f} / M\right) \mathbb{E}\left[\log _{2}\left(1+\bar{P}_{o}\left|\left(\overline{\mathbf{r}}_{\mathrm{MRC}}^{i}\right)^{\dagger} \mathbf{g}_{i}\right|^{2}\right)\right] \\
& =\left(W_{f} / M\right) \log _{2}(e) e^{1 / \bar{P}_{o}} \sum_{k=0}^{M-1} \frac{\Gamma\left(-k, \frac{1}{\bar{P}_{o}}\right)}{\bar{P}_{o}^{k}} .
\end{aligned}
$$

The MRC-based feedback obtains the array gain, but it shows low spectral efficiency due to the orthogonal channel division as $M$ becomes large. The performance of V-MIMO feedback compared with MRC feedback is characterized in the different value of SNR as follows.

- Low SNR: As $\bar{P}_{o} \rightarrow 0$, the number of average feedback bits in MRC based feedback is approximated as $B_{\mathrm{MRC}}^{i} \approx \frac{W_{f} \bar{P}_{o}}{M} \mathbb{E}\left[\left|\left(\overline{\mathbf{r}}_{\mathrm{MRC}}^{i}\right)^{\dagger} \mathbf{g}_{i}\right|^{2}\right] \log _{2} e=W_{f} \bar{P}_{o} \log _{2} e$, while that of ZF based V-MIMO is determined by $B_{\mathrm{ZF}}^{i} \approx \frac{W_{f} \bar{P}_{o}}{M} \mathbb{E}\left[\left|\left(\overline{\mathbf{r}}_{\mathrm{ZF}}^{i}\right)^{\dagger} \mathbf{g}_{i}\right|^{2}\right] \log _{2} e=\frac{W_{f} \bar{P}_{o}}{M} \log _{2} e$. Therefore, the cooperative decoding for CSI feedback under the bandwidth partitioning provides a higher performance gain than the V-MIMO feedback scheme in the low SNR regime, where $B_{\mathrm{MRC}}^{i}=M B_{\mathrm{ZF}}^{i}$.

- High SNR: As $\bar{P}_{o}$ increases, the average feedback bit in MRC based feedback method in (4) is approximated by

$$
\begin{aligned}
B_{\mathrm{MRC}}^{i} & \approx \frac{W_{f}}{M} \cdot \mathbb{E}\left[\log _{2}\left(\bar{P}_{o}\left|\left(\overline{\mathbf{r}}_{\mathrm{MRC}}^{i}\right)^{\dagger} \mathbf{g}_{i}\right|^{2}\right)\right] \\
& =\frac{W_{f}}{M} \cdot\left(\log _{2}\left(\bar{P}_{o}\right)+\mathbb{E}\left[\log _{2} \chi_{2 M}^{2}\right]\right) .
\end{aligned}
$$

Moreover, the average feedback bit in ZF based feedback method in (3) is approximated by

$$
\begin{aligned}
B_{\mathrm{ZF}}^{i} & \approx W_{f} \cdot \mathbb{E}\left[\log _{2}\left(\frac{\bar{P}_{o}}{M}\left|\left(\overline{\mathbf{r}}_{\mathrm{ZF}}^{i}\right)^{\dagger} \mathbf{g}_{i}\right|^{2}\right)\right] \\
& =W_{f} \cdot\left(\log _{2}\left(\frac{\bar{P}_{o}}{M}\right)+\mathbb{E}\left[\log _{2} \chi_{2}^{2}\right]\right)
\end{aligned}
$$

where the $\left|\left(\overline{\mathbf{r}}_{\text {MRC }}^{i}\right)^{\dagger} \mathbf{g}_{i}\right|^{2}$ and $\left|\left(\overline{\mathbf{r}}_{\mathrm{ZF}}^{i}\right)^{\dagger} \mathbf{g}_{i}\right|^{2}$ follow the distribution of $\chi_{2 M}^{2}$ and $\chi_{2}^{2}$, respectively [22].

Therefore, we conclude that $B_{\mathrm{ZF}}^{i}=M B_{\mathrm{MRC}}^{i}$ in the high SNR regime.

Throughout this paper, we consider ZF-based feedback scheme as the suggested V-MIMO feedback scheme, since it provides better performance gain than that of MRC based feedback scheme over the operational threshold (i.e., relatively high SNR).

In a realistic channel model, the number of feedback bits of $\mathbf{h}_{i}$ over instantaneous channel $\mathbf{G}$ is represented as

$$
\bar{B}_{\mathrm{ZF}}^{i}=W_{f} \log _{2}\left(1+\frac{\bar{P}_{o}}{M}\left|\left(\overline{\mathbf{r}}_{\mathrm{ZF}}^{i}\right)^{\dagger} \mathbf{g}_{i}\right|^{2}\right)
$$

where $\bar{B}_{\mathrm{ZF}}^{i}$ is continuously changed in given channel realization. However, adopting instantaneous $\bar{B}_{\mathrm{ZF}}^{i}$-bit quantization at each receiver causes several practical implementation issues due to the additional control signals required for reporting the dynamic quantization level to receivers at every channel realization and multiple channel codebooks for each allocated quantization level. Therefore, the average feedback bits $B_{\mathrm{ZF}}^{i}$ is allocated for each channel $\hat{\mathbf{h}}_{i}$ quantization for considering the feasibility of implementation, which approaches almost the same result as instantaneous $\bar{B}_{\mathrm{ZF}}^{i}$-bit allocation in V-MIMO feedback in cases of large $\bar{P}_{o}$. Note that $\bar{B}_{\mathrm{ZF}}^{i}$ and $B_{\mathrm{ZF}}^{i}$ are approximated as

$$
\begin{aligned}
& \bar{B}_{\mathrm{ZF}}^{i} \approx W_{f}\left(\log _{2}\left(\frac{\bar{P}_{o}}{M}\right)+\log _{2}\left(\left|\left(\overline{\mathbf{r}}_{\mathrm{ZF}}^{i}\right)^{\dagger} \mathbf{g}_{i}\right|\right)\right) \\
& B_{\mathrm{ZF}}^{i} \approx W_{f}\left(\log _{2}\left(\frac{\bar{P}_{o}}{M}\right)+\mathbb{E}\left[\log _{2}\left(\left|\left(\overline{\mathbf{r}}_{\mathrm{ZF}}^{i}\right)^{\dagger} \mathbf{g}_{i}\right|\right)\right]\right) .
\end{aligned}
$$

As shown in (8), the second term is negligible as SNR becomes large so that $B_{\mathrm{ZF}}^{i}=\bar{B}_{\mathrm{ZF}}^{i}$. This approximation is also verified by numerical results in Section 5 . In practice, $\left\lfloor B_{\mathrm{ZF}}^{i}\right\rfloor$ bits are applied for the number of quantized V-MIMO feedbacks since the feedbacks bits should be an integer to design a practical channel codebook, where $\lfloor\cdot\rfloor$ denotes the floor function. 


\subsection{Virtual-MIMO feedback: bandwidth partitioning}

V-MIMO is performed by the following sequential procedures: (1) local data exchange between neighboring transmitters, (2) CSI feedback from the receiver to transmitter, and (3) cooperative transmission from the transmitter cluster to the corresponding receivers. For optimal bandwidth allocation for V-MIMO, it is required for considering both bandwidth for local exchange and data transmission/CSI feedback. However, the corresponding optimization requires the super central-unit having all channel information over the network to control bandwidth allocation, which are impractical in the wireless ad hoc environment. Therefore, we investigate the optimal partition of the bandwidth $W$ for both V-MIMO transmission and feedback assuming that the local data exchange has been ideally performed prior to the data transmission. This assumption is followed by the network model in Section 2, where a local data exchange links between transmitters have much shorter propagation distances than the data transmission link between transmitters and receivers. As a result, the bandwidth required for local data (intra-cluster) exchanges would be much smaller than the bandwidth for long-range (intercluster) since the high-speed channel links are provided between transmitters.

Note that the desired symbol is transmitted over ( $W-$ $W_{f}$ ) and $W_{f}$ is used for CSI feedback from receivers to the corresponding transmitters. Using V-MIMO feedback, the spectral efficiency is represented as

$$
C_{W}=\frac{\left(W-W_{f}\right)}{W} \cdot \mathbb{E}\left[\sum_{i=1}^{M} \log _{2}\left(1+\operatorname{SINR}_{i}\right)\right]
$$

where the signal-to-interference and noise ratio $\left(\mathrm{SINR}_{i}\right)$ is denoted as

$$
\operatorname{SINR}_{i}=\frac{P_{o} / M\left|\mathbf{h}_{i}^{T} \hat{\mathbf{v}}_{i}\right|^{2}}{\left(1+P_{o} / M \sum_{i \neq j}\left|\mathbf{h}_{i}^{T} \hat{\mathbf{v}}_{j}\right|^{2}\right)}
$$

In (10), $\hat{\mathbf{v}}_{i}$ is the transmit beamforming vector for the $i$ th data stream transmission which is determined by using the CSI $\left\{\hat{\mathbf{h}}_{1}, \ldots, \hat{\mathbf{h}}_{M}\right\}$ received in virtual-MIMO feedback with feedback bits of $B_{\mathrm{ZF}}^{i}=W_{f} \cdot R_{m}$. Although it is hard to solve the closed-form of optimal $W_{f}^{*}$ that maximizes (9), we derive the scaling of $W_{f}^{*}$ with system parameters using $\bar{C}_{W}=\frac{\left(W-W_{f}\right)}{W} \cdot \mathbb{E}\left[\sum_{i=1}^{M} \log _{2}\left(\mathrm{SIR}_{i}\right)\right]$, which provides the accurate result when $P_{o}$ is large enough to assume the interference-limited environment. Note that we rewrite $\mathrm{SINR}_{i}$ to $\mathrm{SIR}_{i}$ and upper-bound of the spectral efficiency using Jensen's inequality, for analytical simplicity.

$$
\begin{aligned}
& \bar{C}_{W} \leq \frac{\left(W-W_{f}\right)}{W} \cdot M \log _{2}\left(\mathbb{E}\left[\mathrm{SIR}_{i}\right]\right) \\
& \stackrel{(a)}{\approx} \frac{\left(W-W_{f}\right)}{W} \cdot M \log _{2}\left(2^{\frac{W_{f} R_{m}}{(M-1)}} \cdot \mathbb{E}\left[\frac{\chi_{2}^{2}}{\chi_{2(M-1)}^{2}}\right]\right) \\
& \stackrel{(b)}{=} \frac{\left(W-W_{f}\right)}{W} \cdot M \log _{2}\left(\frac{2^{\frac{W_{f} R_{m}}{(M-1)}}}{M-2}\right)
\end{aligned}
$$

where the distribution of SIR under limited feedback on (a) is derived in [22], (b) follows from the fact that $X=$ $\frac{2(M-1)}{2} \cdot \frac{\chi_{2}^{2}}{\chi_{2(M-1)}^{2}}$ is $F$-distribution with mean $\frac{M-1}{M-2}$ [23], and signal-to-interference ratio $\left(\mathrm{SIR}_{i}\right)$ is denoted as

$$
\operatorname{SIR}_{i}=\frac{P_{o} / M\left|\mathbf{h}_{i}^{T} \hat{\mathbf{v}}_{i}\right|^{2}}{P_{o} / M \sum_{i \neq j}\left|\mathbf{h}_{i}^{T} \hat{\mathbf{v}}_{j}\right|^{2}}
$$

As shown in (11), the spectral efficiency is scaled with $\log _{2}\left(2^{\frac{W_{f} R_{m}}{(M-1)}}\right)$ due to the quantization error while it is also scaled with $\left(W-W_{f}\right)$ with respect to $W_{f}$. Therefore, the choice of $W_{f}$ shows the trade-off between the performance loss due to the quantization error and the increase of transmission spectral efficiency. From (11), the optimal $W_{f}^{*}$ is found by solving $\frac{\partial \bar{C}_{W}}{\partial W_{f}}=0$ where

$$
\frac{\partial \bar{C}_{W}}{\partial W_{f}}=M \log _{2}(M-2)+M\left(-\frac{W_{f} R_{m}}{M-1}\right)
$$

and then we obtain $W_{f}^{*}$ as follows.

$$
W_{f}^{*}=\frac{(M-1) \log _{2}(M-2)}{2 R_{m}}+\frac{W}{2} .
$$

Therefore, the amount of bandwidth for CSI feedback is increased with $\mathcal{O}\left(M \log _{2}(M)\right)$ and inversely proportional to the feedback channel rate $R_{m}$ achieved by V-MIMO feedback.

\section{Virtual-MIMO transmission: cooperative zero-forcing precoding}

In this section, we propose $\mathrm{V}$-MIMO transmission that enables the conventional ZF precoding for cooperative data transmission via the local data exchange and VMIMO feedback proposed in the preceding section. While the Section 3 focused on the optimality of bandwidth between data link and feedback link between transmitter and the receivers, we studied further effects on the local data exchange for constructing V-MIMO transmitters and the analysis on the optimal node clustering to maximize the spectral efficiency throughout this section.

For implementing V-MIMO feedback, it is assumed that the transmitters jointly detect feedback information to improve CSI quality. As illustrated in Fig. 2, the procedure for the V-MIMO transmission can be described by three 

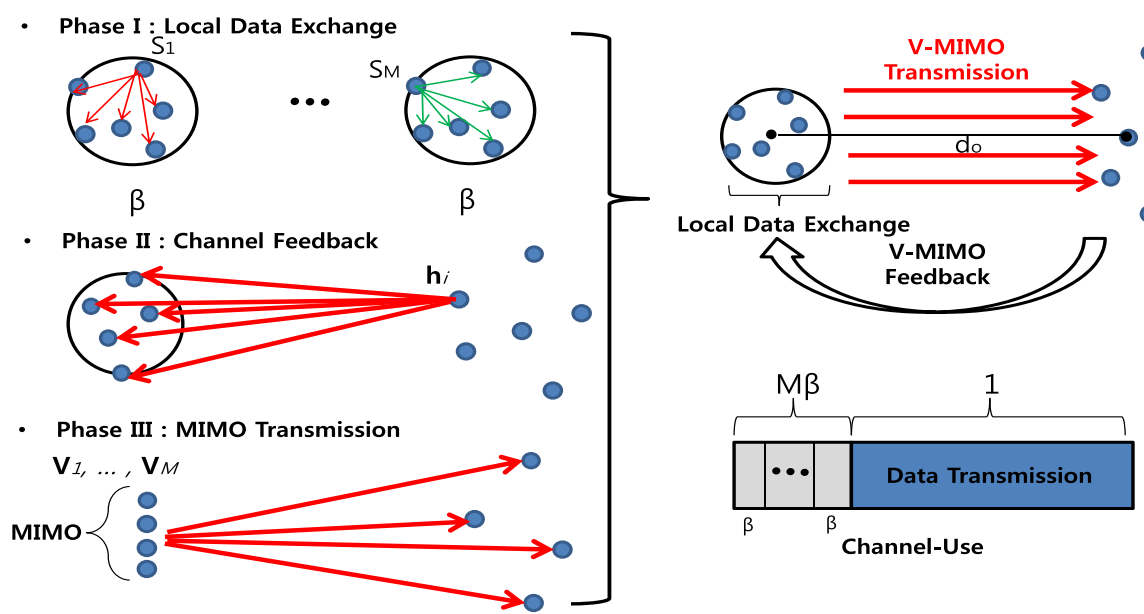

Fig. 2 Virtual-MIMO transmission and feedback

steps which is summarized as follows and analyzed in the subsections.

- Step I: (Local data exchange) The $i$ th transmitter broadcasts its desired signal to the $(M-1)$ neighboring transmitters over $\beta$ bandwidth. Since $M$ data streams are exchanged between nodes over orthogonal bandwidth in FDMA manner, a total of $\beta M$ bandwidth is used for local data exchange, to be transmitted towards the corresponding receivers.

- Step II: (V-MIMO feedback) Prior to the data transmission, each transmitter sends pilot signals to the receiver. Then, each receiver estimates CSI of channel links between all transmitters and the corresponding receiver. Finally, CSI is fed back to the transmitter through the reverse channel. All corresponding CSIs are obtained at all transmitters from joint V-MIMO decoding suggested in Section 3.

- Step III: (V-MIMO transmission) Transmitter clusters design the cooperative ZF precoding to minimize the inter-user interference then communicate with their corresponding receivers.

\subsection{Local data exchange}

Since the achievable data rate between intra-cluster transmitters should be higher than the data rate between transmitter-receiver pairs [16], the following condition is required for performing $\mathrm{V}$-MIMO transmission

$$
\beta \cdot C_{\text {local }} \geq R
$$

where $\beta$ is the bandwidth for local data exchange at each transmitter and $R$ represents the target data rate at the $i$ th transmitter-receiver link. Therefore, the minimum required bandwidth for local data exchange at the $i$ th transmitter is represented as

$$
\beta=\frac{R}{\log _{2}\left(1+\frac{P_{l}}{N_{o}}\right)} .
$$

\subsection{Long-range data transmission}

\subsubsection{Infinite feedback channel}

We consider the case of perfect CSI, corresponding to high-resolution feedback ( $\left.B_{\mathrm{ZF}} \rightarrow \infty\right)$. Assuming that the distances between transmitters and the corresponding receivers are approximately same (or homogeneous), the achievable rate at the $i$ th transmitter-receiver link is represented as (2). However, Due to the discrepancy between the local and long-range channel gain and the fluctuation of small-scale fading between transmitters and receivers, the achievable rate in (2) does not always support the target data rate $R$ at the $i$ th transmitter-receiver link. Therefore, it causes the outage probability of achievable rate so that the outage capacity of $i$ th link is represented as follows.

$$
\begin{aligned}
R_{\mathrm{ZF}, \text { out }}^{i} & =\left(1-P_{\text {out }}\right) \cdot R \\
& =\left(1-P\left(\left|\mathbf{h}_{i} \mathbf{v}_{i}\right|^{2} \leq \frac{M}{P_{o}}\left(2^{R}-1\right)\right)\right) R \\
& =\left(1-P\left(\chi_{2}^{2} \leq \frac{M}{P_{o}}\left(2^{R}-1\right)\right)\right) R \\
& =e^{-\frac{M}{P_{o}}\left(2^{R}-1\right)} R .
\end{aligned}
$$

where $P_{\text {out }}=P\left(R_{Z F}^{i}<R\right)$. Moreover, the $M \beta$ channels are used for the local exchange among $M$ transmitters and thus the spectral efficiency of ZF-based V-MIMO is defined as

$$
R_{\mathrm{ZF}, \text { out }}^{S}=\frac{1}{M \cdot \beta+1} e^{-\frac{M}{P_{o}}\left(2^{R}-1\right)} \cdot R_{\text {sum }}
$$

where $R_{\text {sum }}=M \cdot R$. Since $\beta$ is proportional to $d_{l}$, the spectral efficiency becomes higher when $d_{l}$ becomes 
smaller. Also, large $P_{o}$ increases the spectral efficiency as the outage probability approaches zero.

\subsubsection{Limited feedback channel}

Given $B_{\mathrm{ZF}}^{i}$ feedback bits at each receiver, the residual interference due to the quantization error in ZF precoding is approximated as the sum of $(M-1)$ exponential random variables in [21] and the achievable rate at the $i$-th link is represented as

$$
\begin{aligned}
\hat{R}_{\mathrm{ZF}}^{i} & =\log _{2}\left(1+\frac{P_{o} / M\left|\mathbf{h}_{i}^{T} \hat{\mathbf{v}}_{i}\right|^{2}}{\left(1+P_{o} / M \sum_{i \neq j}\left|\mathbf{h}_{i}^{T} \hat{\mathbf{v}}_{j}\right|^{2}\right)}\right) \\
& =\log _{2}\left(1+\mathrm{SINR}_{i}\right) \\
& \approx \log _{2}\left(1+\frac{\gamma x}{(1+\delta y)}\right)
\end{aligned}
$$

where $\gamma=P_{o} / M, \delta=\frac{P_{o}}{M} \cdot 2^{-\frac{B_{\mathrm{ZF}}}{M-1}}, x \sim \chi_{2}^{2}$ and $y \sim \chi_{2(M-1)}^{2}$. Similar to the case of perfect CSI, the outage capacity of the $i$-th transmitter-receiver link in the limited feedback is derived as follows.

Proposition 1 Given $B_{\mathrm{ZF}}^{i}$ and $P_{o}$, the outage capacity of $Z F$ based V-MIMO transmission is derived as

$$
\hat{R}_{\mathrm{ZF}, \mathrm{out}}^{i}=e^{-\frac{t}{\gamma}} \cdot I_{r} \cdot R
$$

where $t=2^{R}-1$ and $I_{r}=\left(\frac{1}{\delta}\right)^{M-1}\left(\frac{t}{\gamma}+\frac{1}{\delta}\right)^{-(M-1)}$

Proof See Appendix.

From proposition 1, the large number of $M$ degrades the system performance as it increases the outage probability of each transmitter-receiver link. Moreover, $I_{r}$ represents the effect of quantization error due to limited feedback, which follows $I_{r} \leq 1$ in given $B_{\mathrm{ZF}}^{i}$ feedback bits. The degrading factor of the residual interference $I_{r}$ goes to one when $B_{\mathrm{ZF}}^{i}$ is large enough so that the result of (20) approaches the outage capacity in (17).

\subsection{The optimal number of active transmitters}

As the number of nodes participating in V-MIMO transmission increases, the multiplexing gain can be linearly increased with $M$. However, the large number of clustering nodes $M$ also increases both local-exchange overhead and outage probability so that increasing $M$ is not always an optimal solution from the perspective of spectral efficiency since it requires a huge amount of the channel bandwidth for local exchange (see (18)). In this section, we provide the optimal number of nodes that maximizes (18) as follows. To obtain $M^{*}$, we differentiate (18) with $M$ as follows.

$$
\begin{aligned}
\frac{\partial R_{\mathrm{ZF}}^{\mathrm{S}}}{\partial M}= & \frac{\partial}{\partial M}\left(\frac{M R}{M \beta+1}\right) e^{-\frac{M}{P_{O}}\left(2^{R}-1\right)} \\
& +\left(\frac{M R}{M \beta+1}\right) \frac{\partial}{\partial M}\left(e^{-\frac{M}{P_{o}}\left(2^{R}-1\right)}\right) \\
= & \frac{R}{(M \beta+1)^{2}} e^{-\frac{M}{P_{o}}\left(2^{R}-1\right)} \\
& +\frac{M R}{M \beta+1}\left(-\frac{1}{P_{o}}\left(2^{R}-1\right)\right) e^{-\frac{M}{P_{o}}\left(2^{R}-1\right)} \\
= & 0 .
\end{aligned}
$$

Then, (21) is derived as

$$
\beta M^{2}+M-\left(\frac{P_{o}}{2^{R}-1}\right)=0
$$

Solving the above equation with $M>0$, we obtain the following proposition.

Proposition 2 Given $R$ and $P_{o}$, the optimal number of node $M^{*}$ that maximizes the spectral efficiency of (18) is determined as

$$
M^{*}=\frac{\sqrt{1+4 \beta\left(\frac{P_{o}}{2^{R}-1}\right)}-1}{2 \beta} .
$$

To characterize the decision of $M^{*}$ with system parameters, $M^{*}$ can be approximated as

$$
M^{*} \approx \frac{\sqrt{P_{o}}}{\sqrt{\beta\left(2^{R}-1\right)}}
$$

in large $P_{o}$, where the high transmission power and small $R$ reduce the outage probability and increase the number of cooperative nodes, respectively. From the result in proposition 2, we suggest the following clustering operation to maximize the spectral efficiency for V-MIMO transmission.

- $M^{*}$ activate node selection: note that $M$ nodes are distributed over transmitter cluster, where $M^{*}<M$. The node selection of $M^{*}$ of $M$ is performed by the round-robin manner, where it provides the fairness of data transmission opportunity, implementable with low complexity. In a round-robin approach, the selected nodes give a priority of data transmission to other idle nodes in a next transmission phase.

- V-MIMO transmission for $M^{*}$ : the selected $M^{*}$ transmitters only share the desired data streams $\left\{s_{1}, s_{2}, \ldots, s_{M^{*}}\right\}$ over the $M^{*} \beta$ bandwidth as each of the selected $M^{*}$ nodes broadcasts its own data in $\beta$ bandwidth. Then, $\left(M-M^{*}\right)$ idle nodes, which are 
not allowed to transmit their own data, can help improving the achievable rate in (2) by providing array gain (see Fig. 3). In that case, $\left|\mathbf{h}_{i} \mathbf{v}_{i}\right|^{2}$ follows a chi-square distribution with DoF of $2\left(M-M^{*}+1\right)$; then, the outage capacity is given by

$$
\begin{aligned}
\tilde{R}_{\mathrm{opt}}^{i} & =\left(1-P_{\mathrm{out}}\right) \cdot R \\
& =\left(1-P\left(\left|\mathbf{h}_{i} \mathbf{v}_{i}\right|^{2} \leq t\right)\right) \cdot R \\
& =\left(1-P\left(\chi_{2\left(M-M^{*}+1\right)}^{2} \leq t\right)\right) \cdot R \\
& =1-\left(1-e^{-t} \sum_{i=1}^{M-M^{*}+1} \frac{t^{i-1}}{(i-1) !}\right) \cdot R \\
& =e^{-t} \sum_{i=1}^{M-M^{*}+1} \frac{t^{i-1}}{(i-1) !} \cdot R .
\end{aligned}
$$

where $t=\frac{M^{*}}{P_{o}}\left(2^{R}-1\right)$. Therefore, the large number of idle nodes can reduce the outage probability and $\tilde{R}_{\text {opt }}^{i}$ approaches $R$ as $M$ goes to infinity.

\section{Simulation results}

In this section, the analysis of proposed V-MIMO feedback and transmission schemes and the corresponding performance gains are demonstrated by simulation results. Firstly, we show that the average feedback bit allocation over V-MIMO feedback channel approaches the same result as instantaneous feedback bit allocation in certain SNR regimes. Figure 4 depicts the relative difference between $B_{\mathrm{ZF}}^{i}$ and $\bar{B}_{\mathrm{ZF}}^{i}$ in (3) and (7), where $\xi=$ $\frac{\left|\bar{B}_{\mathrm{ZF}}^{i}-B_{\mathrm{ZF}}^{i}\right|}{B_{\mathrm{ZF}}^{i}}$. As the transmission power of feedback channel, $\bar{P}_{o}$, increases, $\xi$ converges to 0 and then it becomes smaller than 0.1 when $\bar{P}_{o}>12 \mathrm{~dB}$. Therefore, using the average V-MIMO feedback bits in the medium range of $\bar{P}_{o}$,

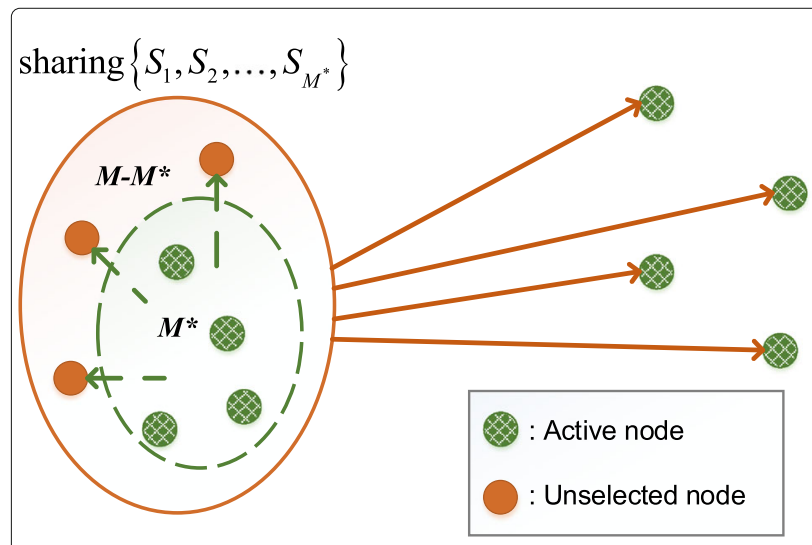

Fig. 3 Virtual-MIMO transmission in $M^{*} \leq M$

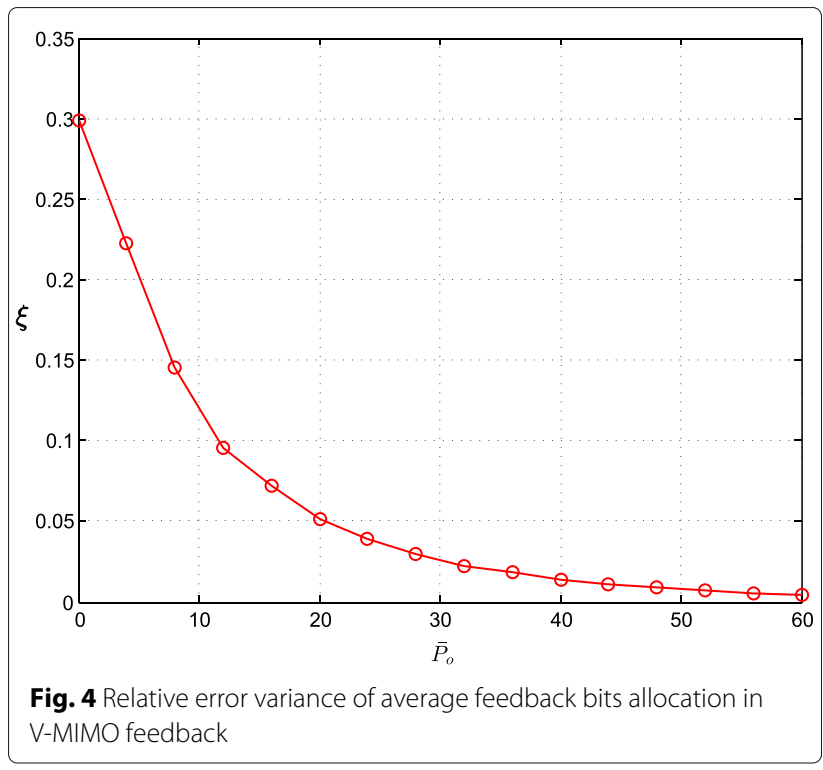

such as 10-15 dB, guarantees a marginal performance loss compared with the use of instantaneous feedback bits so that constant V-MIMO feedback bits computed in average sense does not provide the significant performance loss rather than the use of variable feedback bits from instantaneous channel.

Figure 5 compares the throughput of ZF precoding given the average feedback bits and instantaneous feedback bits in V-MIMO feedback. In the ergodic sense, we show that the sum throughput using instantaneous VMIMO feedback bits is highly close to that of average (equally allocated) V-MIMO feedback bits given $\bar{P}_{o}=$ 10 and $15 \mathrm{~dB}$. Moreover, the performance of proposed V-MIMO feedback and that of conventional feedback

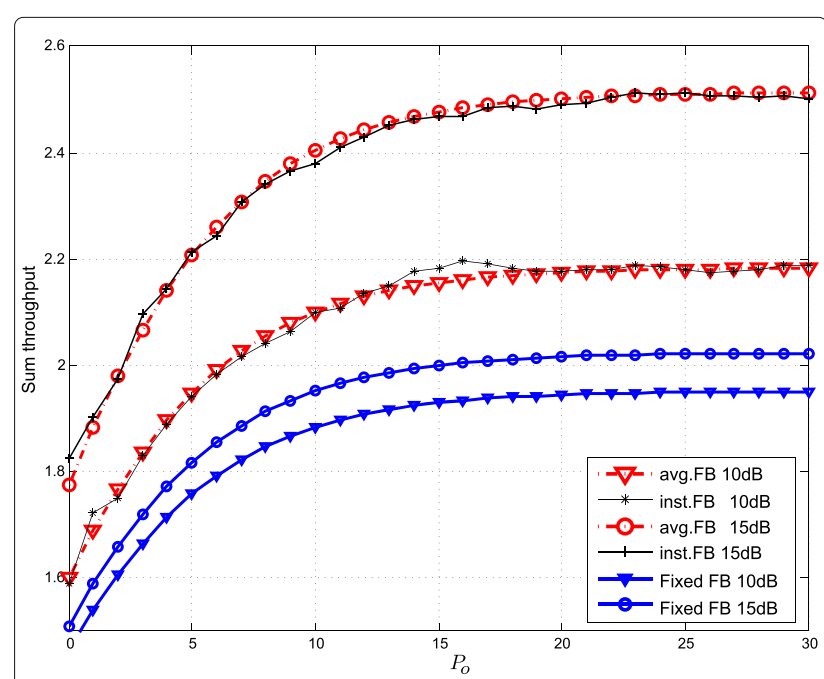

Fig. 5 Comparison of sum throughput of the cooperative ZF precoding in averaged and instantaneous V-MIMO feedback bits 
approach is compared, where the conventional feedback method considers the equal feedback bits for each receiver under the orthogonal feedback bandwidth. On the saturation region in high SNR, the 25 and $15 \%$ of performance gain are shown in the proposed feedback method compared with the conventional method at $\bar{P}_{o}=10$ and 15 $\mathrm{dB}$, respectively.

Given $W=50, P_{o}=30 \mathrm{~dB}$, and $\left(d_{l} / d_{o}\right)=0.3$, Figs. 6 and 7 compare the spectral efficiency of ZF precoding in (9) under the MRC and ZF based V-MIMO feedback schemes. In Fig. 6, it is shown that the performance gain of ZF V-MIMO feedback over MRC feedback increases as $\bar{P}_{o}=7,10$, and $13 \mathrm{~dB}$ when $M=5$. However, the performance of MRC based CSI feedback is rather better than the ZF V-MIMO feedback in the low feedback power such as $\bar{P}_{o}=7 \mathrm{~dB}$. In Fig. 7 , the optimal resource partitioning based on (14) is plotted given $\bar{P}_{o}=10 \mathrm{~dB}$ and the different value of $M$. As derived in (14), the optimal bandwidth allocation for CSI feedback increases with $M$ and the analytical results match the simulations.

In Fig. 8, the spectral efficiency of ZF based V-MIMO transmission is compared in the distributed network, where $P_{o}=20 \mathrm{~dB}, R=2,\left(d_{l} / d_{o}\right)=0.3$, and $W_{f}=30$ with the transmission power of feedback channel, respectively. As shown in (20), the performance gain of ZF transmission in large $\mathrm{M}$ generates the strong residual interference given the finite rate feedback channel. The efficiency of ZF transmission of V-MIMO feedback approaches that of perfect CSI as $\bar{P}_{o}$ goes to large. Furthermore, the gain of ZF V-MIMO feedback provides a dramatic increase of system performance than the MRC-based CSI feedback in large $\bar{P}_{o}$ since it achieves the full multiplexing gain for CSI acquisition. Fig. 9 shows the spectral efficiency of ZF based V-MIMO transmission and its optimal $M^{*}$. As

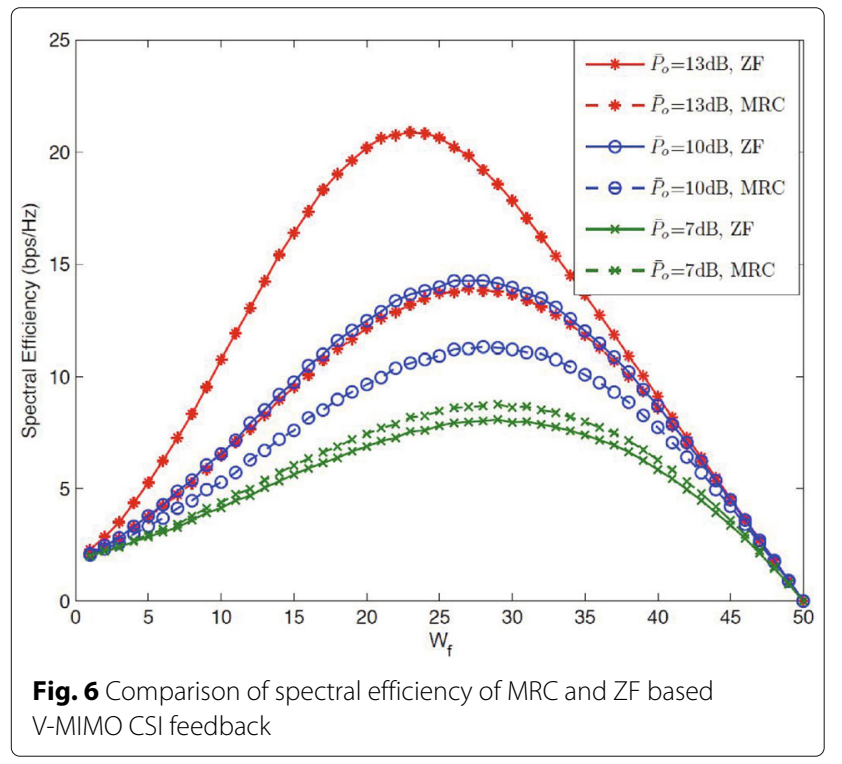

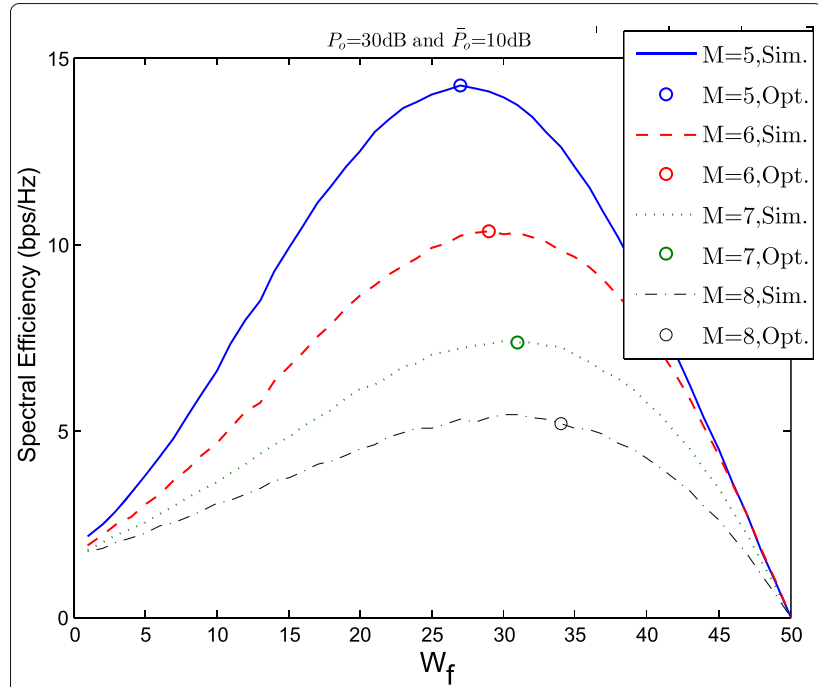

Fig. 7 Optimal resource partitioning for V-MIMO feedback in the different number of cooperative nodes

derived in (23), the optimal solution in high $P_{o}$ is that all nodes participate in V-MIMO, while the corresponding solution becomes non-cooperative when $P_{o}$ approaches low SNR region. However, in the medium value of transmission power, increasing the number of cooperation nodes does not always provide a higher spectral efficiency as it requires a large amount of bandwidth for local data exchange at the transmitter cluster. Setting the parameters $P_{o}=10 \mathrm{~dB}, R=1$, and $\left(d_{l} / d_{o}\right)=0.1,0.3$, and 0.6 , the transmitters and receivers are located within the distance of $d_{l}$, respectively. Then, we compare the curves of spectral efficiency and analytical results in (23). While the increase of cooperative nodes provides a higher multiplex-

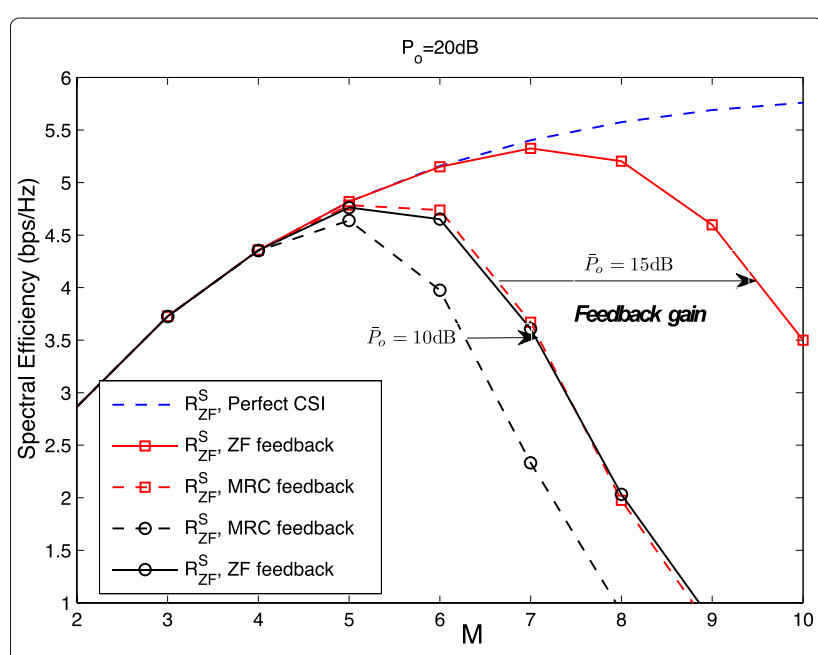

Fig. 8 Spectral efficiency of cooperative ZF transmission in limited feedback 


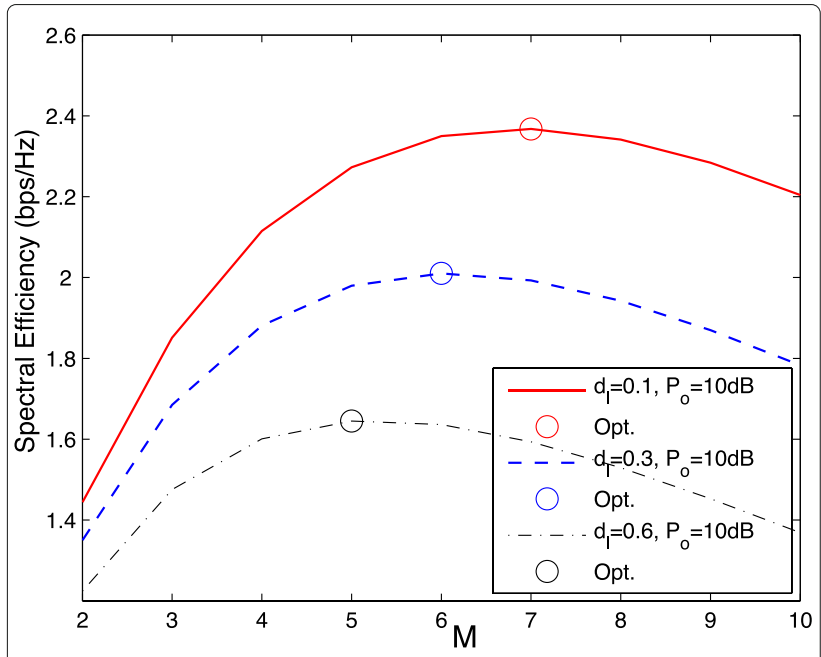

Fig. 9 Comparison of ZF precoding and the optimal number of nodes

ing gain, it also requires additional costs for local data exchange, as shown in (18). Therefore, the proposed VMIMO is more beneficial for the case of i) short distance between neighboring transmitters which provides highspeed local data link and ii) the short distance between the corresponding receivers that experiences a strong interuser interference channel, respectively.

\section{Conclusions}

In this paper, an efficient feedback and data transmission scheme using V-MIMO has been proposed in a wireless ad hoc network. We show that the cooperative feedback improves the accuracy of CSI compared with the orthogonal channel feedback and provides abundant channel resources for data transmission by reducing the use of channel resource for CSI feedback. Given the proposed feedback scheme, the outage capacity of ZF based V-MIMO transmission under the transmitter cooperation is analyzed and the optimal number of active nodes that maximize the spectral efficiency is proposed for $\mathrm{V}$ MIMO based clustering. Future works will examine the design of V-MIMO feedback and transmission in randomly distributed transmitter and receiver nodes and propose hierarchical clustering and cooperation with VMIMO feedback in the existence of multiple transmitter and receiver clusters. Moreover, we leave the analysis on stochastic geometric approach under V-MIMO system, where it can represent the effectiveness of the proposed algorithms on more realistic environment.

\section{Endnote}

${ }^{1}$ The usage of codebook designs exploiting the channel statistics, such as [24, 25], can improve the system performance in V-MIMO transmission.

\section{Appendix}

\section{Proof of proposition 1}

Note that the outage probability of ZF precoding at the $i$-th transmitter-receiver pair is $P\left(R_{\mathrm{ZF}}^{i}<R\right)=P\left(\mathrm{SINR}_{i}\right.$ $<t$ ), where $t=2^{R}-1$. Then, the outage probability is derived as

$$
\begin{aligned}
& P\left(\mathrm{SINR}_{\mathrm{i}}<t\right)=P\left(\frac{\gamma x}{1+\delta y}<t\right) \\
& =P\left(x<\frac{t(1+\delta y)}{\gamma}\right) \\
& =\int_{0}^{\infty} \int_{0}^{\frac{t(1+\delta y)}{\gamma}} p_{X}(x) p_{Y}(y) d x d y \\
& =\int_{0}^{\infty}\left[1-e^{-\frac{t}{\gamma}(1+\delta y)}\right] p_{Y}(y) d y \\
& =1-\int_{0}^{\infty} e^{-\frac{t}{\gamma}(1+\delta y)} p_{Y}(y) d y \\
& =1-\frac{e^{-t / \gamma}}{\Gamma(M-1)} \int_{0}^{\infty} e^{-\left(\frac{t \delta}{\gamma}+1\right) y} y^{M-2} d y \\
& =1-e^{-\frac{t}{\gamma}\left(\frac{1}{\delta}\right)^{M-1}}\left(\frac{t}{\gamma}+\frac{1}{\delta}\right)^{-(M-1)} .
\end{aligned}
$$

Therefore, the outage capacity at the $i$-th transmitterreceiver pair is given as

$$
\begin{aligned}
R_{\mathrm{ZF}, \text { out }}^{i} & =\left(1-P_{\text {out }}\right) R \\
& =e^{-\frac{t}{\gamma}}\left(\frac{1}{\delta}\right)^{M-1}\left(\frac{t}{\gamma}+\frac{1}{\delta}\right)^{-(M-1)} R .
\end{aligned}
$$

\section{Acknowledgements}

This research was supported by ICT R\&D program of MSIP/IITP. (No. B0126-15-1012, Multiple access technique with ultra-low latency and high efficiency for tactile internet services in loT environments)

\section{Competing interests}

The authors declare that they have no competing interests.

\section{Author details}

${ }^{1}$ School of Electrical and Electronics Engineering, Yonsei University, Seoul, South Korea. ${ }^{2}$ Department of Electrical and Electronics Engineering, The

University of Hong Kong, Pok Fu Lam, Hong Kong.

Received: 19 October 2015 Accepted: 17 October 2016

Published online: 04 November 2016

References

1. IE Telatar, Capacity of multi-antenna Gaussian channels.Euro. Trans. Telecom. 10(6), 585-595 (1999)

2. A Paulraj, R Nabar, D Gore, Introduction to Space-Time Wireless Communications. (Cambridge University Press, Cambridge, 2003)

3. G Caire, S Shamai, On the achievable throughput of a multiantenna Gaussian broadcast channel. IEEE Trans. Inform. Theory. 49(7), 1691-1706 (2003)

4. N Jindal, MIMO broadcast channels with finite-rate feedback. IEEE Trans. Inform. Theory. 52(11), 5045-5060 (2006)

5. M Kobayashi, N Jindal, G Caire, Training and feedback optimization for multiuser MIMO downlink. IEEE Trans. Commun. 59(8), 2228-2240 (2011)

6. N Ravindran, $\mathrm{N}$ Jindal, Limited feedback-based block diagonalization for the MIMO broadcast channel. IEEE J. Sel. Areas in Commun. 26(8), 1473-1482 (2008) 
7. P Viswanath, DNC Tse, R Laroia, Opportunistic beamforming using dumb antennas. IEEE Trans. Inform. Theory. 48(6), 1277-1294 (2002)

8. J Zhang, R Chen, JG Andrews, A Ghosh. RW Heath Jr., Networked MIMO with clustered linear precoding. IEEE Trans. Wirel. Commun. 8(4), 1910-1921 (2009)

9. D Gesbert, S Hanly, H Huang, S Shamai, O Simeone, Yu W, Multi-cell MIMO cooperative networks: a new look at interference. IEEE J. Sel. Areas Commun. 28(9), 1380-1408 (2010)

10. X Li, M Chen, W Liu, Application of STBC-encoded cooperative transmissions in wireless sensor networks. IEEE Signal Process. Lett. 12(2), 134-137 (2005)

11. SK Jayaweera, Virtual-MIMO-based cooperative communication for energy-constrained wireless sensor networks. IEEE Trans. Wirel. Commun. 5(5), 984-989 (2006)

12. Z Ding, WH Chin, KK Leung, Distributed beamforming and power allocation for cooperative networks. IEEE Tran. Wirel. Commun. 7(5), 1817-1822 (2008)

13. R Mudumbai, DR Brown, U Madhow, HV Poor, Distributed transmit beamforming: challenges and recent progress. IEEE Commun. Magazine. 47(2), 102-110 (2009)

14. M Hong, Z Xu, M Razaviyayn, Z-Q Luo, Joint user grouping and linear virtual beamforming: complexity, algorithms and approximation bounds. IEEE J. Sel. Areas Commun. 31(10), 2013-2027 (2013)

15. SH Seyedmehdi, G Boudreau, An efficient clustering algorithm for device-to-device assisted virtual MIMO. IEEE Trans. Wirel. Commun. 13(3), 1334-1343 (2014)

16. AD Coso, U Spagnolini, C Ibars, Cooperative distributed MIMO channels in wireless sensor networks. IEEE J. Sel. Areas Commun. 25(2), 401-414 (2007)

17. L Dong, AP Petropulu, HV Poor, Weighted cross-layer cooperative beamforming for wireless networks. IEEE Trans. Sig. Process. 57(8), 3240-3252 (2009)

18. J Park, S Lee, $M^{2}-m^{2}$ beamforming for virtual-MIMO broadcasting in multi-hop relay networks. IEEE J. Sel. Areas Commun. 30(8), 1358-1369 (2012)

19. D Cassioli, MZ Win, AF Molisch, The ultra-wide bandwidth indoor channel: from statistical model to simulations. IEEE. J. Sel. Areas Commu. 20(6), 1247-1257 (2002)

20. TYoo, A Goldsmith, On the optimality of multiantenna broadcast scheduling using zero-forcing beamforming. IEEE J. Sel. Areas Commun. 24(3), 528-541 (2006)

21. J Zhang, M Kountouris, JG Andrews. IEEE Trans. Commun. 59(3), 803-814 (2011)

22. J Zhang, JG Andrews, Adaptive spatial intercell interference cancellation in multicell wireless networks. IEEE J. Sel. Areas Commun. 28(9), 1455-1468 (2010)

23. A Papoulis, SU Pillai, Probability, random variables and stochastic processes, 4th. (McGraw-Hill Education, New York, 2002), p. 208

24. Y Cheng, VKN Lau, Y Long, A scalable limited feedback design for network MIMO using per-cell product codebook. IEEE Trans. Wirel. Commun. 9(10), 3093-3099 (2010)

25. JH Lee, W Choi, Unified codebook design for vector channel quantization in MIMO broadcast channels. IEEE Trans. Sig. Process. 63(10), 2509-2519 (2015)

\section{Submit your manuscript to a SpringerOpen ${ }^{\circ}$ journal and benefit from:}

- Convenient online submission

$\checkmark$ Rigorous peer review

- Immediate publication on acceptance

- Open access: articles freely available online

- High visibility within the field

- Retaining the copyright to your article

Submit your next manuscript at $\boldsymbol{\nabla}$ springeropen.com 